\title{
IMAGE RECOVERY FROM IRREGULARLY LOCATED SPECTRAL SAMPLES
}

\author{
P.J. Bones, N.D. Blakeley* \\ University of Canterbury \\ Dept. Electrical and Electronic Engineering \\ Christchurch, New Zealand
}

\author{
R.P. Millane $e^{\dagger}$ \\ Purdue University \\ Computational Sci. and Eng. Program \\ West Lafayette, Indiana
}

\begin{abstract}
Recovery of magnetic resonance images from irregular sampling sets is investigated from the point of view of moment discretization of the Fredholm equation of the first kind. The limited spatial extent of the object is known a priori and the sampling schemes considered each have mean density lower than that imposed by the Nyquist limit. The recovery formula obtained has the same form as a standard irregular sampling technique. A practical means of performing the recovery for very large data sets by utilising the block Toeplitz structure of the matrix involved is presented.
\end{abstract}

\section{INTRODUCTION}

Recovery of a 2-D signal (image) from a finite set of irregularly spaced spectral samples is a problem that arises in fields such as aperture-synthesis radio telescopy [1] and magnetic resonance imaging [2]. Clearly, if the samples can be arranged to lie on a regular grid and certain other conditions are met, the WhittakerKotel'nikov-Shannon sampling theorem [3] may be applied, and an image straightforwardly reconstructed via the inverse discrete Fourier transform.

In radio telescopy, however, the locations of spectral samples are fixed by the physical siting of antennae and by the rotation of the earth. The process known as "gridding" has been commonly used to approximate the spectrum on a regular grid from the irregular set of measured samples and the iterative CLEAN algorithm has also been popular [1]. The success of the latter method relies to some extent on prior knowledge that stellar objects tend to be compact and isolated.

In magnetic resonance (MR) imaging, it is possible to position samples in the spatial frequency domain ( $k$-space) virtually at will [2]. A common approach is to obtain measurements on a recti-linear grid, allowing efficient image reconstruction via the inverse fast Fourier transform (IFFT). However, to reduce the time to gather sufficient data to form an image (and thus to save expensive scanner time or to follow real-time events), as few spectral samples as possible should be gathered, perhaps resulting in an irregular set of samples. For example, spiral sampling is an effective way of traversing $k$-space [2], with samples taken along a spiral trajectory commencing at the spectral origin. Another possibility is not to measure some elements of the recti-linear data (in order to reduce the scan time). An alternative to the IFFT is thus required.

${ }^{*}$ N.D. Blakeley is the recipient of a Bright Futures doctoral scholarship from the Foundation of Research, Science and Technology, New Zealand.

${ }^{\dagger}$ R.P. Millane was supported by a NSF International Cooperatice Science Program Grant (INT-9904481).
Marks suggests why it should be possible to effect recovery from sample sets that are a subset of the Nyquist set [4]: if the replicated versions of the signal in the spatial domain exhibit gaps, then the Nyquist samples are not independent, and those not measured (or 'missing') may be recovered from those remaining. In MR imaging, these gaps occur since the object being imaged is of limited spatial extent (the cross-section of a patient's head, for example); the object is nonzero only inside the region of support (ROS).

The 1-D irregular sampling problem was first addressed by Yen [5], and the interpolation formula he derived has been subsequently rediscovered from several different approaches and its performance under noise analysed $[6,7,8]$. In contrast, the 2-D problem is not as extensively studied $[9,10]$. In Section 2 , we pose the problem of MR image recovery from irregularly located spectral samples, and derive a reconstruction formula that has the same form as Yen's. Methods and practical issues are discussed in Section 3, with results shown in Section 4.

\section{THEORY}

The MR imaging problem may be posed as the reconstruction of a continuous object, $f(\mathbf{r})$, from a set of measurements taken in $k$ space, $g\left(\mathbf{k}_{n}\right)$, where $\mathbf{k}_{n} \in \mathcal{D}$, a set of $N$ locations in $k$-space [2]. A Fourier transform relationship exists between $f(\mathbf{r})$ and $g\left(\mathbf{k}_{n}\right)$ :

$$
g\left(\mathbf{k}_{n}\right)=\int_{\mathcal{S}} f(\mathbf{r}) K\left(\mathbf{k}_{n}, \mathbf{r}\right) d \mathbf{r},
$$

where $\mathcal{S}$ is the ROS and $K(\mathbf{k}, \mathbf{r})=\exp (-j 2 \pi \mathbf{k} \cdot \mathbf{r})$

Many problems in image recovery are characterised by a Fredholm equation of the first kind [11], and inspection of Eq.(1) reveals that it is in fact a moment discretized version $[12,13]$. We define the operator $T_{N}: L_{2}(\mathcal{S}) \rightarrow \mathbb{C}^{N}$ by

$$
T_{N} f=\left[\begin{array}{c}
\left\langle f(\mathbf{r}), \bar{K}\left(\mathbf{k}_{1}, \mathbf{r}\right)\right\rangle \\
\left\langle f(\mathbf{r}), \bar{K}\left(\mathbf{k}_{2}, \mathbf{r}\right)\right\rangle \\
\vdots \\
\left\langle f(\mathbf{r}), \bar{K}\left(\mathbf{k}_{N}, \mathbf{r}\right)\right\rangle
\end{array}\right] \text { and } g=\left[\begin{array}{c}
g\left(\mathbf{k}_{1}\right) \\
g\left(\mathbf{k}_{2}\right) \\
\vdots \\
g\left(\mathbf{k}_{N}\right)
\end{array}\right]
$$

where $\langle\cdot, \cdot\rangle$ denotes the inner product in $L_{2}(\mathcal{S})$ and the bar denotes conjugation.

Eq.(1) may now be written in the form $T_{N} f=g$, and the unique least-squares solution is given by $[12,13]$

$$
\hat{f}(\mathbf{r})=T_{N}^{+} g=\sum_{n=1}^{N} b_{n} \bar{K}\left(\mathbf{k}_{n}, \mathbf{r}\right), \quad \mathbf{r} \in \mathcal{S},
$$


where $T_{N}^{+}$is the Moore-Penrose inverse of $T_{N}$ and $\left\{b_{n}\right\}_{n=1}^{N}$ is a set of scalar coefficients (discussed below).

If $g(\mathbf{k})$ is a member of the range of the original infinite-dimensional operator, $K$, then $\hat{f}$ can be written explicitly as

$$
\hat{f}(\mathbf{r})=\sum_{m=1}^{N} \sum_{n=1}^{N} g\left(\mathbf{k}_{m}\right) \gamma_{m n} \bar{K}\left(\mathbf{k}_{n}, \mathbf{r}\right), \quad \mathbf{r} \in \mathcal{S}
$$

where $\gamma_{m n}$ are elements of the Moore-Penrose inverse of the $N \times N$ matrix $Q$ :

$$
\begin{aligned}
Q_{m n} & =\left\langle K\left(\mathbf{k}_{n}, \mathbf{r}\right), K\left(\mathbf{k}_{m}, \mathbf{r}\right)\right\rangle \\
& =\int_{\mathcal{S}} K\left(\mathbf{k}_{n}, \mathbf{r}\right) \bar{K}\left(\mathbf{k}_{m}, \mathbf{r}\right) d \mathbf{r} .
\end{aligned}
$$

Furthermore, if the set $\left\{K\left(\mathbf{k}_{n}, \mathbf{r}\right)\right\}_{n=1}^{N}$ is linearly independent then $\gamma_{m n}$ may instead be defined as elements of the inverse of $Q$ and the set $\left\{b_{n}\right\}_{n=1}^{N}$ of Eq.(2) is uniquely determined by solving the matrix equation $Q b=g$, where $b=\left[b_{1} b_{2} \ldots b_{N}\right]^{T}$ and ${ }^{T}$ denotes transpose.

Eq.(3) is the interpolation formula for the irregular sampling problem originally devised by Yen [5]. There are, however, two main differences: firstly, the sampling is performed in the spatial frequency domain, and secondly the solution is obtained in the 'other' domain (i.e. that in which sampling is not performed)

\section{METHODS}

Several practical issues need addressing in the implementation of Eq.(3). Firstly, the large number of measurements involved results in an ill-conditioned algebraic system to solve [12]. The presence of noise on the measurements exacerbates the problem. In [7], the use of Miller regularization was suggested for matrix $Q$; that is, a small constant is added to the diagonal of the matrix prior to inversion. In fact, under additive white Gaussian noise, the least-squares estimator, the maximum likelihood estimator, and the maximum a posteriori probability estimator were all shown to be the Miller-regularized Yen interpolation algorithm. Furthermore, in [8] it was shown that for additive white noise, Miller regularization resulted from a statistical analysis, and the magnitude of the regularization parameter should be chosen according to the variance of the white noise. Even without noise, the roundoff error in the numerical calculations may be regarded as white noise.

Alternatively, the method of singular value decomposition (SVD) may be used to pseudo-invert the matrix $[7,14]$. This has the advantage of allowing the basis functions corresponding to the singular values that are too inaccurate to be useful (i.e. those closest to zero) to be left out altogether. Finally, the iterative conjugate gradient method [15] may be used to solve for the coefficient set $\left\{b_{n}\right\}_{n=1}^{N}$, with the inherent regularization properties of the algorithm [16] providing a more stable solution. Moreover, instead of an inverse or pseudo-inverse required to be found, matrix multiplication is the most computationally expensive operation, which may be performed via a fast algorithm under certain conditions.

The derivation of the elements of matrix $Q$ in Eq.(4) is based on the object's region of support. A rectangular region is commonly used $[6,14]$ resulting in a sinc function, but better results should be obtained from a support that better describes the object. All the results presented in this paper use a modified version of the Shepp and Logan head phantom [17], which is made up of ten ellipses, as shown in Fig. 1(a). The region of support may clearly be taken to be the outer ellipse, and while in this case the chosen support region is tight, it is envisaged that in practice an ellipse could be constructed to loosely fit an arbitrary head shape. The benefit of using the head phantom is that the object and its spectrum may be described analytically (see [17]), providing a good simulation of actual MR imaging data while maintaining experimental flexibility.

Implicit in Eq.(4) is the notion that the support region is a mask with value either one or zero. In [8], it is argued that if the statistics of the object are known they should be incorporated; for example, a Gaussian autocovariance models extreme smoothness and continuity. With our problem, however, it is not clear how to incorporate any additional information without biasing the recovery (the large discontinuity at the edge of the skull clearly presents a difficulty). problem:

Finally, we give the specific recovery formulae for the MR

$$
\begin{aligned}
\hat{f}(\mathbf{r}) & =\sum_{n=1}^{N} b_{n} \exp \left(j 2 \pi \mathbf{k}_{n} \cdot \mathbf{r}\right), \quad \mathbf{r} \in \mathcal{S} \\
Q_{m n} & =\int_{\mathcal{S}} \exp \left(j 2 \pi\left(\mathbf{k}_{m}-\mathbf{k}_{n}\right) \cdot \mathbf{r}\right) d \mathbf{r} .
\end{aligned}
$$

Note that the value of $Q_{m n}$ is simply the inverse Fourier transform of the support region evaluated at location $\mathbf{k}_{m}-\mathbf{k}_{\boldsymbol{n}}$. A convenient solution method is to first solve the matrix equation $Q b=g$ and then use the result to apply Eq.(5).

\section{RESULTS}

We consider two types of irregular sampling, both of which provide a speed advantage over standard recti-linear sampling in the acquisition of MR images. The first of these is a spiral trajectory, commencing at the origin and designed to fill a certain circular region of $k$-space. The second is a standard recti-linear grid, except that some lines of samples are not measured. The motivation for this scheme is that in practice a line of $k$-space is read as a single unit, so a speed advantage is only realised if entire lines are not measured. This is an example of the 'missing samples' problem addressed in [4], but here it is being approached from an irregular sampling point of view.

It should be noted that in using a recti-linear data set, advantage may be taken of the fact that the object being imaged is real, and therefore that its spectral samples exhibit Hermitian symmetry. This is exploited by imaging only half of $k$-space, or slightly more than half to account for any phase irregularities introduced by the scanning process [2]. The sampling strategy we are suggesting here is fully compatible with this existing technique.

The results presented come from three sampling sets. For the first set, $\mathcal{D}_{1}$, we started with a $64 \times 64$ recti-linear grid and removed eight lines at random (giving a $12.5 \%$ speed increase over the full grid), yielding a total of 3,584 samples. This produces a matrix that is near the largest that may be inverted readily using our computer equipment. The second set, $\mathcal{D}_{2}$, also has 3,584 samples, and comes from a spiral trajectory chosen to cover approximately the same area of $k$-space as $\mathcal{D}_{1}$. Finally $\mathcal{D}_{3}$ contains 14,336 samples and was created from a $128 \times 128$ recti-linear grid with sixteen lines removed at random (again giving a $12.5 \%$ speed increase). The matrix produced by $\mathcal{D}_{3}$ was too large to invert, and even troublesome to create. However, the form of $\mathcal{D}_{3}$ generates a matrix that has block Toeplitz structure, allowing the matrix equation to be solved using the conjugate gradient method with 
the matrix multiplication at each iteration sped up by utilising the FFT [18]; the complete matrix need not be explicitly created at all.

Fig. 1 gives benchmarks against which to compare the recovered images: the continuous head phantom test object is shown in (a), while (b) shows recovery from a full $64 \times 64$ recti-linear data set via IFFT and (c) demonstrates the result if IFFT recovery from $\mathcal{D}_{1}$ is attempted with the missing lines set to zero. In other words, (a) shows the ideal recovery, (b) gives the standard IFFT recovery from the given region of $k$-space, and (c) shows the effect the missing data has on corrupting the image. For comparison with Fig. 2, the spectra for (b) and (c) were zeropadded prior to inverse transformation to produce reconstructions on $256 \times 256$ grids. It should be noted that the Gibbs' ringing artefacts in (b) are due to truncation of the spectral data obtained (i.e. only a finite number of samples are collected), while the aliasing artefacts in (c) are due to undersampling.

Fig. 2(a), (b) and (c) show recovery from $\mathcal{D}_{1}, \mathcal{D}_{2}$ and $\mathcal{D}_{3}$, respectively, in the absence of noise, while (d), (e) and (f) show the recovery after white Gaussian noise was added to the respective spectral measurements for the equivalent of $20 \mathrm{~dB}$ SNR. In (a) and (b), recovery was performed using matrix inversion with Miller regularization, with the parameter set to an arbitrary small value. In (d) and (e), the conjugate gradient method was used, since under noise it was found that better results were obtained in this manner. The conjugate gradient method was also used for (c) and (f), but taking advantage of the block Toeplitz structure. In the cases employing conjugate gradient, a straightforward algorithm was used; pre-conditioning and early termination should allow even better results to be obtained.

While the recovery formula used (Eq.(5)) produces a continuous object, in order to display the results here all reconstructions have been made onto a $256 \times 256$ grid in the spatial domain.

\section{CONCLUSIONS}

The problem of recovery from irregularly located spectral samples may be investigated from the point of view of moment discretization of the Fredholm equation of the first kind. The recovery formula obtained has the same form as the standard irregular sampling technique due originally to Yen. In practice, difficulties may arise in performing the numerical calculations, both in terms of stability of the solution and tractability. For the sampling set based on a recti-linear grid, a practical means for dealing with very large data sets was established utilising the block Toeplitz structure of the matrix. Both sampling types presented provide a decrease in acquisition time over conventional recti-linear sampling, and recovery was shown to be good even in the presence of noise.

\section{REFERENCES}

[1] N.W. Brouw, "Aperture synthesis," in Methods in Computational Physics, B. Alder, S. Fernbach, and M. Rotenberg, Eds., vol. 14, pp. 131-175. Academic Press, 1975.

[2] Z.P. Liang and P.C. Lauterbur, Principles of Magnetic Resonance Imaging: A Signal Processing Perspective, IEEE Press, New York, USA, 2000.

[3] Abdul J. Jerri, "The Shannon sampling theorem-its various extensions and applications: a tutorial review," Proc. IEEE, vol. 65, no. 11, pp. 1565-1597, Nov. 1977.
[4] R.J. Marks Il, "Multidimensional-signal sample dependency at Nyquist densities," J. Opt. Soc. Amer. A, vol. 3, no. 2, pp. 268-273, Feb. 1986

[5] J.L. Yen, "On nonuniform sampling of bandwidth-limited signals," IRE Trans. Circuit Theory, vol. 3, pp. 251-257, 1956.

[6] D.S. Chen and J.P. Allebach, "Analysis of error in reconstruction of two-dimensional signals from irregularly spaced samples," IEEE Trans. Acoust., Speech, and Signal Process., vol. ASSP-35, no. 2, pp. 173-180, Feb. 1987.

[7] Duncan J. Wingham, "The reconstruction of a band-limited function and its Fourier transform from a finite number of samples at arbitrary locations by singular value decomposition," IEEE Trans. Signal Processing, vol. 40, no. 3, pp. 559-570, Mar. 1992.

[8] H. Choi and D.C. Munson, Jr., "Stochastic formulation of bandlimited signal interpolation," IEEE Trans. Circuits and Systems-II: Analog and Digital Signal Process., vol. 47, no. 1, pp. 82-85, Jan. 2000.

[9] K.D. Sauer and J.P. Allebach, "Iterative reconstruction of band-limited images from nonuniformly spaced samples," IEEE Trans. Circuits and Systems, vol. CAS-34, no. 12, pp. 1497-1506, Dec. 1987

[10] T. Strohmer, "Computationally attractive reconstruction of bandlimited images from irregular samples," IEEE Trans. Image Processing, vol. 6, no. 4, pp. 540-548, Apr. 1997.

[11] E.R. Pike, J.G. McWhirter, M. Bertero, and C. de Mol, "Generalised information theory for inverse problems in signal processing," IEE Proc., Pt. F, vol. 131, no. 6, pp. 660-667, Oct. 1984.

[12] C.K. Rushforth, "Signal restoration, functional analysis, and Fredholm integral equations of the first kind," in Image Recovery: Theory and Application, H. Stark, Ed., pp. 1-27. Academic Press, Orlando, Florida, USA, 1987.

[13] M.Z. Nashed, "On moment-discretization and least-squares solutions of linear integral equations of the first kind," $J$. Math. Anal. Appl., vol. 53, pp. 359-366, 1976.

[14] R. Van de Walle, H.H. Barrett, K.J. Myers, M.I. Altbach, B. Desplanques, A.F. Gmitro, J. Cornelis, and I. Lemahieu, "Reconstruction of MR images from data acquired on a general nonregular grid by pseudoinverse calculation," IEEE Trans. Medical Imaging, vol. 19, no. 12, pp. 1160-1167, Dec. 2000.

[15] David G. Luenberger, Linear and Nonlinear Programming, Addison-Wesley, Reading, Mass., USA, 2nd edition, 1984.

[16] M. Bertero and P. Boccacci, Introduction to inverse problems in imaging, Institute of Physics Publications, Bristol, UK, 1998.

[17] S.X. Pan and Avinash C. Kak, "A computational study of reconstruction algorithms for diffraction tomography: interpolation versus filtered backpropagation," IEEE Trans. Acoust., Speech, and Signal Process., vol. ASSP-31, no. 5, pp. 12621275, Oct. 1983.

[18] G. Strang, "A proposal for Toeplitz matrix calculations," Stud. Appl. Math., vol. 74, pp. 171-176, 1986. 


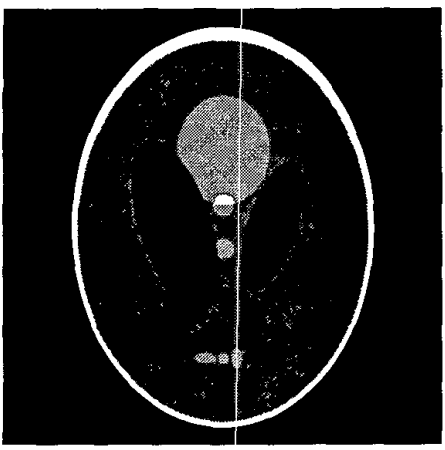

(a) test object

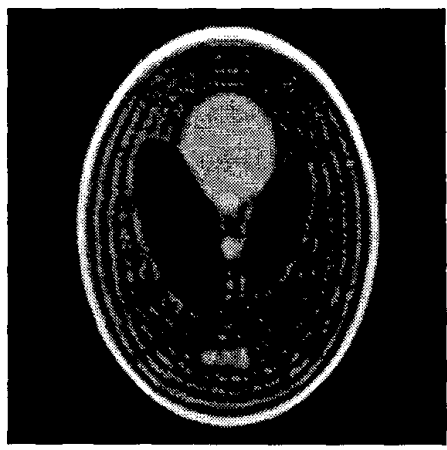

(b) full data IFFT recovery

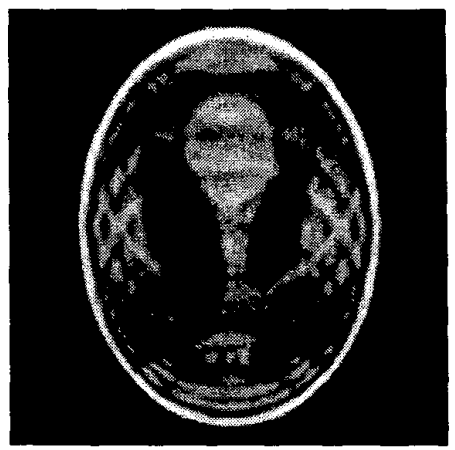

(c) missing data IFFT recovery

Fig. 1. Benchmarks for the recovered images: (a) the head phantom test object, (b) IFFT recovery from full $64 \times 64$ recti-linear grid, and (c) IFFT recovery from $\mathcal{D}_{1}$ with missing data set to zero.

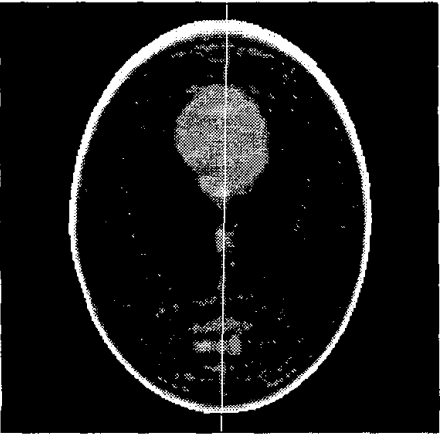

(a) $\mathcal{D}_{1}$, no noise

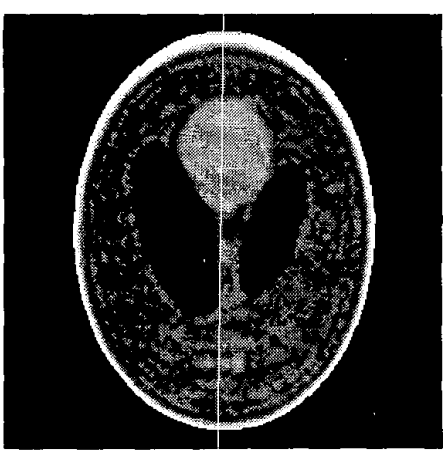

(d) $\mathcal{D}_{1}$, with noise

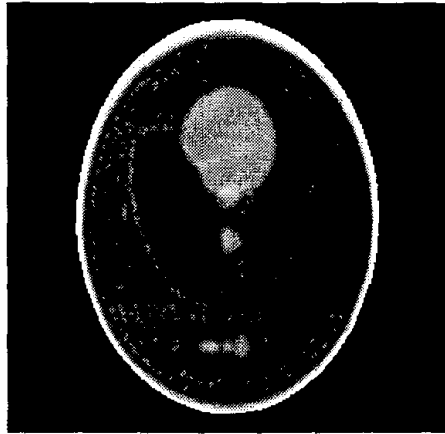

(b) $\mathcal{D}_{2}$, no noise

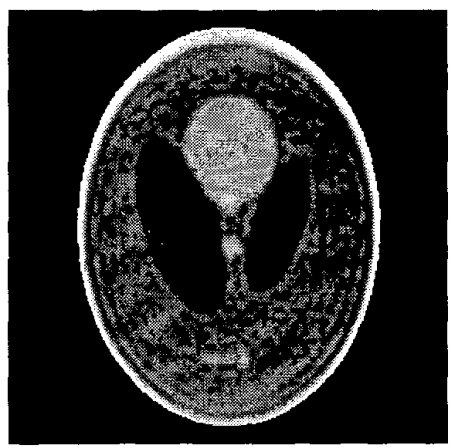

(e) $\mathcal{D}_{2}$, with noise

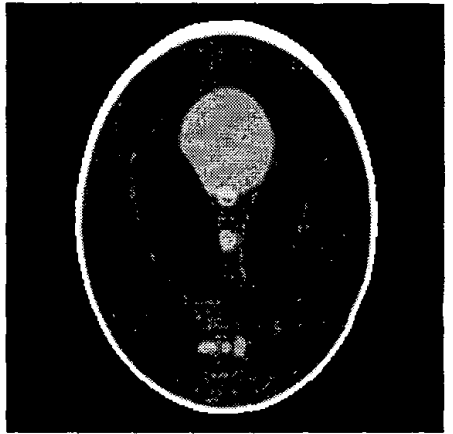

(c) $\mathcal{D}_{3}$, no noise

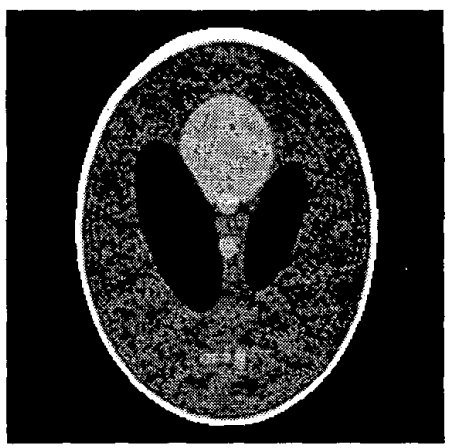

(f) $\mathcal{D}_{3}$, with noise

Fig. 2. Recovery of the head phantom test object from $\mathcal{D}_{1}, \mathcal{D}_{2}$ and $\mathcal{D}_{3}$, with and without noise equivalent to $20 \mathrm{~dB}$ SNR. 